\title{
The impact of triptan and doxycycline on neuroinflammatory biomarkers in acute migraine: study protocol of a randomized controlled trial

\section{Abstract}

Background: Increased inflammatory cytokines and matrix metalloproteinases (MMPs) have been recently implicated in migraine. Inflammation may be a key player in the pathophysiology of migraine by altering blood-brain barrier (BBB) function. As an inflammation induced MMP, MMP-9 is involved in both BBB disruption and neuropathic pain, and is largely derived by neutrophil degranulation during neutrophil-BBB interaction. The tetracycline group of antibiotics may suppress MMP production and neutrophil degranulation.

Methods/Design: We hypothesize that migraine is a primary neuroinflammatory disorder. We will address this theory by measuring serum biomarkers in healthy controls and after one of three abortive therapy strategies for acute migraine attacks and inter-ictally. We intend to investigate known neuroinflammatory biomarkers with a focus on BBB breakdown during acute migraine attacks and assess marker responses to conventional treatment (triptans), novel MMP targeted therapy (doxycycline), or a combination of triptan and doxycycline. We will measure the effects of the interventions on neuroinflammatory markers for acute migraine attacks, clinical outcomes (headache relief and recurrence) for acute migraine attacks, interictal neuroinflammatory load per serum biomarkers in migraineurs versus healthy controls, and neuroinflammatory markers correlation with headache duration and peak headache intensity during acute migraine attacks.
Shaheen E Lakhan, MD, PhD, MEd, MS ${ }^{1}$

1 Neurological Institute, Cleveland Clinic, Euclid Ave, S100C, Cleveland OH

Contact information:

Shaheen E Lakhan, MD, PhD, MEd, MS. Neurological Institute, Cleveland Clinic, 9500 Euclid Ave, S100C, Cleveland OH 44195.

Tel: (216) 444-2200

” slakhan@gnif.org 
Discussion: To our knowledge, this trial will be the first systematic study on the use of a MMP inhibitor in episodic migraine. Our pilot project data will supplement future projects investigating novel therapeutic strategies such as MMP inhibitors in both migraine acute treatment and prevention.

Trial Registration: ClinicalTrials.gov NCT01653522, registered 07/23/2012.

\section{Background}

Migraine is a common neurovascular disorder that affects over 30 million Americans. There is significant economic impact secondary to headacherelated disability [1]. Current theories of migraine pathophysiology include cortical spreading depression (CSD) and neurogenic inflammation [2]. More recently, a compromised $\mathrm{BBB}$ as a result of neurogenic inflammation has been implicated in migraine pathophysiology [3].

The BBB protects the brain against compounds and pathogens in the blood stream and maintains central nervous system homeostatsis. This neurovascular interface is composed of endothelial tight junctions, basal lamina, and astrocyte endfeet in the brain parenchyma. It is hypothesized that vascular inflammation is a common final pathway for migraine with or without aura [4-8]. A few studies have indicated that inflammatory cytokines are elevated in migraine patients intra- and inter-ictally [9-13]. CSD may create BBB permeability in migraine mediated by MMPs and other inflammatory molecules.

MMPs and tissue inhibitors of MMPs (TIMPS) are involved in the remodeling of extracellular matrix via chemokines and cellular adhesion molecules. An alteration of MMP expression may lead to the breakdown of the BBB, demyelination, cytokine production and an inflammatory response, deposition of amyloid proteins, and tumor invasion and metastasis [14-18]. In migraine, post-headache levels of
MMP-9 were significantly higher than controls [1922]. MMP-9 potentiates chemokines for leukocyteendothelial cell interaction leading to an inflammation cascade at the brain vasculature. The inflammation may be a reason for migraine patients being at a higher risk of stroke [8]. Utilizing anti-inflammatory and -MMP effect, doxycycline coupled with interferon beta-1a reduced multiple sclerosis lesions in humans [23] and minocycline prevented hemorrhagic transformation in acute ischemic strokes in a rate model treated with IV tPA [24]. However, the origin of MMP-9 in migraine patients remains unknown, as does the effect of acute migraine treatment targeting MMP-9.

Compounds such as triptans and magnesium may not cross an intact BBB, however, their utility in migraine may be in the setting of BBB disruption. Moreover, glucocorticoids, commonly used in acute migraine treatment, are known to stabilize the BBB. In a migraine animal model, MMP-9 was downregulated with methyl-prednisolone [25].

Interestingly, Todd Rozen of the Geisinger Medical Group has written on doxycycline use as an antiinflammatory drug for primary headache disorders, especially new daily persistent headache. However, he only published one abstract on his open label use [26], no peer-reviewed paper, and referred to it in a recent review [27]. 


\section{Methods}

\section{Objectives}

Primary endpoint: Determine the effects of oral triptan, doxycycline, and triptan + doxycycline on neuroinflammatory markers for acute migraine attacks.

Secondary endpoints:

1. Compare the effects of oral triptan, doxycycline, and triptan + doxycycline on clinical outcomes (headache relief and recurrence) for acute migraine attacks.

2. Determine if inter-ictal neuroinflammatory load per serum biomarkers is greater in migraineurs than healthy controls.

3. Determine if the levels of neuroinflammatory markers correlate with headache duration and peak headache intensity during acute migraine attacks.

\section{Design}

This is a single site, randomized, 4 arm open-label pilot study of levels of neuroinflammatory markers in adult episodic migraineurs and healthy controls without migraine. We will recruit participants at a major 1,300-bed tertiary teaching hospital, the Cleveland Clinic.

We will recruit two groups of patients to participate in this study: (1) generally healthy adult episodic migraine patients of the Headache Center (the "Actives") or (2) healthy controls who are patients (the "Controls"). The "actives" will have migraine with or without aura that fulfill the 2nd Edition of The International Headache Classification (ICHD-II) criteria for episodic migraine. Migraine recruits ("actives") will also have an active prescription for an oral triptan medication to abort acute migraines. The "actives"/migraineurs will not be on a tetracycline group or other anti-inflammatory medication for the preceding three months. Informed consent will be obtained from all subjects prior to research enrollment and subsequent research bloodwork, diary use, and medication use.
There will be three treatment arms:

1. Oral triptan (as prescribed)

2. Doxycycline (one dose of $200 \mathrm{mg}$ by mouth)

3. Triptan (as prescribed) + Doxycycline (one dose of 200mg by mouth)

There will be one non-treatment arm of "control" subjects who do not have migraines, will not complete a diary, and will have one set of pain free bloodwork drawn.

Subjects will be randomly assigned to one of the three treatment groups until there are about 10 subjects in each group. Venous blood during an inter-ictal period will be drawn at least seven days after the most recent migraine attack. Subjects will be telephoned three days after this bloodwork to document that they have not experienced a migraine since the bloodwork was obtained. The subjects will be instructed to maintain a headache diary containing the following details: (A) date/time of migraine onset, (B) migraine pain intensity based on a four-point scale $(0=$ no pain, $1=$ mild pain, $2=$ moderate pain, $3=$ severe pain); (C) date/time migraine treatment medication was taken including medication name and total dose taken; (D) date/time of migraine resolution and (E) date/time of start and time of stop for any adverse drug reactions and any treatments for those adverse reactions.

The "active" subjects will be instructed to selfadminister oral medication during a migraine attack when the pain level is of moderate to severe intensity. Venous blood will be collected at a designed lab at a six hour timepoint starting from the time migraine treatment is taken.

Both the "active" subjects and the "controls" will also have an inter-ictal (or pain free time for the controls) venous blood obtained at a designed lab. (The "controls"/healthy volunteers will be subjects without a headache disorder who are age and gender matched to the "active" migraineurs in the treatment arms.) Both sets of labs will be delivered to the processing lab within one hour of being drawn, for handling and storage. Specifically 
the specimens will be centrifuged, pipetted into aliquots, labeled, and those sera will be kept at $-80^{\circ} \mathrm{C}$ until further processing is performed. Serum levels of MMP-9 (active enzyme), hs-CRP, TNF-alpha, IL-1 and IL- 6 will be measured by ELISA. Once these study assays are completed and documented, the remaining specimens, if any, will be stored 1 year maximally and maybe used for other institutionally approved studies related to migraine. After 1 year blood samples will be destroyed.

\section{Subject Selection Sample Size}

As this is a pilot study, no formal sample size justification is presented. A descriptive statistical analysis is provided and seems warranted given the goals of the study.

This study will enroll 40 subjects for participation. There are no other sites for this study. 30 subjects will be qualified by whether they meet diagnostic criteria for episodic migraine with or without aura as defined by the ICHD-II and identified as the "actives". The remaining 10 subjects will be healthy volunteers who do not have migraine diagnosis and will be identified as the "controls".

Additionally the actives will have a current triptan prescription, not be pregnant, and not on a medication in the tetracycline group or other antinflammatory medication in the 3 months prior to enrollment.

In the event that an enrolled "active" subject does not treat a qualifying migraine within 6 weeks from enrollment, they will be provided an additional diary to continue documentation each day of any headache activity which they might experience. They would be expected to remain in the study until they have treated a migraine and had their 6 hour blood specimen collected, post-dose.

\section{Inclusion Criteria}

A migraine subject ("active") will be eligible to participate in this study if all of the following criteria apply:
1. subject is 18 years or older at screening

2. subject has been diagnosed with episodic migraine by the ICHD-II criteria

3. subject is not pregnant or nursing

4. subject has not taken a medication in the tetracycline group or anti-inflammatory medication within the prior 3 months to screening

A healthy subject ("control") will be eligible to participate in this study if all of the following criteria apply:

1. subject is 18 year or older at screening

2. subject does not have migraine diagnosis

3. subject is not pregnant or nursing

4. subject has not taken a medication in the tetracycline group or anti-inflammatory medication within the prior 3 months to screening.

\section{Exclusion Criteria}

A subject will not be eligible to participate in this study if any of the following criteria apply:

1. subject is less than 18 years of age at screening

2. subject does not have episodic migraine diagnosis by the ICHD-II criteria

3. subject is pregnant or nursing

4. subject has taken a medication in the tetracycline group or antinflammatory medication within the prior 3 months to screening

A randomization schedule will be used to make the treatment assignments for the study "active" subjects.

\section{Study Dosage Regimen Treatment}

Subjects in the "active" arms will be informed of their treatment randomization assignment at the screening and enrollment visit. The subjects will be instructed to self-administer the assigned oral medication when the migraine pain is moderate to severe in intensity and they are able to return to the designed lab at 6 hours after dosing, in order to have their blood drawn. 
In the event that an "active" subject experiences a migraine on the weekend or at a time when they will not be able to return to the Center for Pain to have their 6 hour post-dose blood draw obtained, they will be instructed to treat that migraine with their usual treatment and wait for the next migraine and use the study drug assignment if that is a viable migraine and timepoint. The "active" subjects would document all migraines they experience on the daily diary.

\section{Rescue Treatment}

Because a primary objective of this study is to obtain venous blood specimens at 6 hours post dose, subjects will be asked to not take additional treatments prior to having this specimen obtained.

At 6 hours post-dose, if the subject has headache pain such that additional treatment is desired, and the subject has had the 6 hour post-dose blood specimen obtained, the subject will be permitted to take a second dose of their assigned treatment or in the case of the subject who is assigned to doxycycline alone, the subject may treat with the usual triptan.

\section{Study Drug Supply}

For those subjects who are randomized to the doxycycline alone treatment arm or the doxycycline and triptan treatment arm, the doxycycline will be provided at the screening and enrollment visit.

- Treatment Assignment will be made according to the randomization schedule of the study.

- Treatment will be open label.

- For study product accountability, subjects will have documented dispensation of study product and will return study drug containers when they present for the 6 hour post-dose blood draw.

- Subjects will record treatment use in the study diary for the treated migraine attack.

- Study drug supply will be procured, stored, labeled and dispensed to the site.

\section{Study Flowsheet}

See Table 1 for the study flowsheet (time and events) listing the study activities throughout the protocol.

Table 1: Study flowchart.

\begin{tabular}{|c|c|c|c|}
\hline & $\begin{array}{c}\text { Screen and } \\
\text { Enrollment } \\
\text { Visit } \\
\text { "Actives") }\end{array}$ & $\begin{array}{c}\text { Screen and } \\
\text { Enrollment } \\
\text { Visit } \\
\text { ("Controls") }\end{array}$ & $\begin{array}{l}6 \text { Hour } \\
\text { Post-Dose } \\
\text { Visit }\end{array}$ \\
\hline \multicolumn{4}{|l|}{ Procedure } \\
\hline $\begin{array}{l}\text { Informed } \\
\text { Consent }\end{array}$ & $x$ & $x$ & \\
\hline $\begin{array}{l}\text { Inclusion/ } \\
\text { Exclusion }\end{array}$ & $x$ & $x$ & \\
\hline Randomization & $x$ & & \\
\hline $\begin{array}{l}\text { Study Drug } \\
\text { Dispensing }\end{array}$ & $x$ & & \\
\hline $\begin{array}{l}\text { Diary } \\
\text { Instruction and } \\
\text { Dispensing }\end{array}$ & $X$ & & \\
\hline $\begin{array}{l}\text { Urine Pregnancy } \\
\text { Test }\end{array}$ & $x$ & & \\
\hline $\begin{array}{l}\text { Study Drug } \\
\text { collection }\end{array}$ & & & $x$ \\
\hline Diary Collection & & & $x$ \\
\hline $\begin{array}{l}\text { Phone Call at } 3 \\
\text { Days After Pain } \\
\text { Free Draw }\end{array}$ & $x$ & $x$ & \\
\hline $\begin{array}{l}\text { Pain Free Blood } \\
\text { Draw }\end{array}$ & $x$ & $x$ & \\
\hline $\begin{array}{l}6 \text { Hour Post- } \\
\text { Dose Blood } \\
\text { Draw }\end{array}$ & & & $x$ \\
\hline
\end{tabular}

\section{Safety}

Adverse events will be collected via subject report on study diaries and at the blood draw visits. These adverse events will be noted in the electronic medical record and will be noted from the date of consent signing until the second blood draw is completed and the study diary is returned.

\section{Data Analysis}

According to the literature (19-22), the 95\% confidence interval for the difference in means of MMP-9 
between the migraine group and the control group will be approximately $50 \pm 30 \mathrm{ng} / \mathrm{mL}$. It is expected that the confidence intervals for the inter-ictal and post-treatment differences in the treated group will be considerably narrower due to the elimination of patient-to-patient variation when analyzing our primary endpoint. We will also compare clinical outcomes in the three treatment arms, but our planning statistics will not be powered for this analysis as a primary outcome measure.

Individual variables and differences between pre and post-treatment will be described with means and standard deviation (SD) or medians and interquartile range (IQR). Depending on the distribution, parametric or nonparametric confidence intervals will be constructed for the difference between cases and controls and for the paired differences between inter-ictal and post-treatment in the case group. JMP 9.0 (SAS Institute) will be used to carry out the analyses.

\section{Data Confidentiality}

Subject data points will be collected using the data from the subject diary and the specimen assays. Each enrolled subject will be assigned a study ID number which will be used on the diary pages and the specimen tubes. A master list identifying each subject and their respective ID number will be maintained in the study regulatory files. The data analysis will all be done with de-identified data. The persons with access to the study data would be the study investigators and all data will be maintained online using the hospital mainframe which is password protected.

\section{Ethics and Dissemination}

Ethics approval has been obtained from the Institutional Review Board of the Cleveland Clinic (IRB \#12-061).

\section{Discussion}

We hypothesize that migraine is a primary neuroinflammatory disorder. We will address this theory by measuring serum biomarkers in healthy controls and after one of three abortive therapy strategies for acute migraine attacks and inter-ictally. Moreover, our pilot project will serve as a foundation for further research into neuroinflammatory markers as a response to novel therapeutic agents. To our knowledge, this would be the first systematic study on MMP inhibition for episodic migraine.

\section{Trial Status}

Ongoing, entering recruitment-phase.

\section{List of abbreviations}

BBB: blood-brain barrier; ICHD-II: 2nd Edition of The International Headache Classification; MMP: matrix metalloproteinase; SD: standard deviation; TIMP: tissue inhibitors of matrix metalloproteinases.

\section{Competing Interests}

The author declares that he has no competing interests.

\section{Author's contributions}

SEL: conception and design of the study, data collection, manuscript writing, critical revision, and final approval of the manuscript.

\section{Acknowledgements}

We wish to thank the Cleveland Clinic Neurological Institute, the funding agency.

\section{References}

1. Burton WN, Landy SH, Downs KE, et al. The Impact of Migraine and the Effect of Migraine Treatment on Workplace Productivity in the United States and Suggestions for Future Research. Mayo Clin Proc 2009; 84(5): 436-445. 
2. Longoni M, Ferrarese C. Inflammation and excitotoxicity: role in migraine pathogenesis. Neurol Sci 2006; 27 Suppl 2:S107-10.

3. Pietrobon D, Moskowitz MA. Pathophysiology of migraine. Annu Rev Physiol. 2013; 75: 365-91.

4. Bolay $H$, Reuter $U$, Dunn AK, et al. Intrinsic brain activity triggers trigeminal meningeal afferents in migraine model. Nat Med 2002; 8(2): 136-42

5. Waeber C, Moskowitz MA. Migraine as an inflammatory disorder. Neurology 2005; 64(10 Suppl 2): S9-15.

6. Moskowitz MA, Macfarlane R. Neurovascular and molecular mechanisms in migraine headaches. Cerebrovasc Brain Metab Rev 1993, 5(3): 159-77.

7. Welch KM, Brandes AW, Salerno L, et al. C-reactive protein may be increased in migraine patients who present with complex clinical features. Headache 2006; 46(2): 197-9.

8. Welch KM. Stroke and migraine-the spectrum of cause and effect. Func Neurol 2003; 18(3): 121-6.

9. Koçer A, Memişoğullari $R$, Domaç FM, et al. IL-6 levels in migraine patients receiving topiramate. Pain Pract 2009; 9(5): 375-9.

10. Kaciński M, Gergont A, Kubik A, et al. Proinflammatory cytokines in children with migraine with or without aura. Przegl Lek 2005; 62(11): 1276-80.

11. Gergont A, Kaciński M. Blood interleukin-6 level in children with idiopathic headaches. Neurol Neurochir Pol 2005; 39(4 Suppl 1): S1-8

12. Perini F, D'Andrea G, Galloni E, et al. Plasma cytokine levels in migraineurs and controls. Headache 2005; 45(7): 926-31.

13. Fidan I, Yüksel S, Ymir T, et al. The importance of cytokines, chemokines and nitric oxide in pathophysiology of migraine. J Neuroimmunol 2006; 171(1-2): 184-8.

14. Gupta VK. CSD, BBB and MMP-9 elevations: animal experiments versus clinical phenomena in migraine. Expert Rev Neurother 2009; 9(11): 1595-614

15. Matrisian LM. Metalloproteinases and their inhibitors in matrix remodelling. Trends Genet 1990; 121-125.

16. Chandler S, Miller KM, Clements JM, et al. Matrix metalloproteinases, tumor necrosis factor and multiple sclerosis: An overview. J Neuroimmunol 1997; 72: 155-161.

17. Leppert D, Waubant E, Galardy R, et al. T cell gelatinases mediate basement membrane transmigration in vitro. J Immunol 1995; 154: 4397-4389.

18. Aoki T, Sumii T, Mori T, et al. Blood-brain barrier disruption and matrix-metalloproteinase-9 expression during reperfusion injury: Mechanical versus embolic focal ischemia in spontaneously hypertensive rats. Stroke 2002; 33: 2711-2777.

19. Leira R, Sobrino $T$, Rodríguez-Yáñez $M$, et al. MMP-9 Immunoreactivity in Acute Migraine. Headache 2007; 47(5): 698-702.

20. Imamura K, Takeshima T, Fusayasu E, et al. Increased Plasma Matrix Metalloproteinase-9 Levels in Migraineurs. Headache 2008; 48(1): 135-139.
21. Ashina M, Tvedskov JF, Lipka K, et al. Matrix metalloproteinases during and outside of migraine attacks without aura. Cephalalgia 2010; 30(3): 303-310.

22. Berneckera $C$, Pailera $S$, Kieslinger $P$, et al. Increased matrix metalloproteinase activity is associated with migraine and migraine-related metabolic dysfunctions. European Journal of Neurology 2011; 18: 571-576.

23. Minagar A., Alexander JS., Schwendimann RN., et al, Combination Therapy With Interferon Beta-1a and Doxycycline in Multiple Sclerosis. Arch Neurol 2008; 65(2): 199-204.

24. Machado LS, Sazonova I, Kozak A, et al. Minocycline and tissue plasminogen activator for stroke: Assessment of interaction potential. Stroke 2009; 40(9): 3028-3033.

25. Kim GM, Jin K-S, Chung CS. Differential Effects of Corticosteroids on The Expression of Cyclooxygenase-2, Tumour Necrosis FactorAlpha and Matrix Metalloproteinase-9 in An Animal Model of Migraine. Cephalalgia 2008, 28, 1179-1187.

26. Rozen TD. Doxycycline for treatment resistant new daily persistant headache. Neurology 2008; 70 (Suppl 1): A348 (abstract)

27. Rozen TD. New Daily Persistent Headache: Clinical Perspective. Headache 2011; 51: 650-653.

\section{Comment on this article:}

\section{(f) $[$ in $8+\mathbf{S} P$}

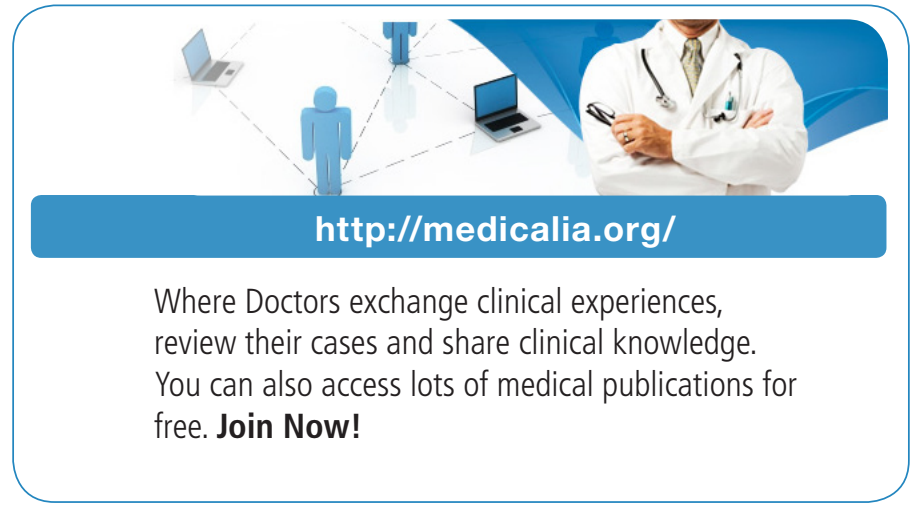

Publish with iMedPub

\section{http://www.imed.pub}

International Archives of Medicine is an open access journal publishing articles encompassing all aspects of medical science and clinical practice. IAM is considered a megajournal with independent sections on all areas of medicine. IAM is a really international journal with authors and board members from all around the world. The journal is widely indexed and classified Q1 in category Medicine. 